\title{
Stochastic Phase Oscillators and Circadian Bioluminescence Recordings
}

\author{
J. ROUGEMONT* AND F. NAEF*广 \\ *Swiss Institute of Bioinformatics, CH-1015 Lausanne, Switzerland; ${ }^{\dagger}$ Swiss Institute of Experimental Cancer \\ Research, Ecole Polytechnique Fédérale de Lausanne, CH-1015 Lausanne, Switzerland
}

\begin{abstract}
Cultured circadian oscillators from peripheral tissues were recently shown to be both cell-autonomous and self-sustained. Therefore, the dominant cause for amplitude reduction observed in bioluminescence recordings of cultured fibroblasts is desynchronization, rather than the damping of individual oscillators. Here, we review a generic model for quantifying luminescence signals from biochemical oscillators, based on noisy-phase oscillators. Our model incorporates three essential features of circadian clocks: the stability of the limit cycle, fluctuations, and intercellular coupling. The model is then used to analyze bioluminescence recordings from immortalized and primary fibroblasts. Fits to population recordings allow simultaneous estimation of the stability of the limit cycle (or equivalently, the stiffness of individual frequencies), the period dispersion, and the interaction strength between cells. Consistent with other work, coupling is found to be weak and insufficient to synchronize cells. Interestingly, we find that frequency fluctuations remain correlated for longer periods than one clock cycle, which is confirmed from individual cell recordings. We discuss briefly how to link the generic model with more microscopic models, which suggests mechanisms by which circadian oscillators resist fluctuations and maintain accurate timing in the periphery.
\end{abstract}

\section{INTRODUCTION}

The reciprocal interactions among environment, genes, and behavior that lead living organisms to evolve circadian clocks are inevitably very complex. Therefore, the attempt to use mathematics to model these phenomena may seem like a daunting enterprise. Yet, circadian biology is one of the earliest fields that has attracted modelers and continues to do so at an ever-increasing rate. One of the reasons why theoreticians have been attracted to the problem is that oscillatory trajectories can be abstracted by a few informative variables, e.g., phase and amplitude. While providing a coarser-grained description, a number of generally applicable concepts can then be explored, e.g., the measurement of phase-response curves to probe the structure of the underlying limit cycles. In this context, the theoretical prediction and subsequent measurement of phase singularities in hatching flies (Winfree 2001) provide a fascinating demonstration of the power of such approaches.

Although these earlier studies were guided by geometric or topological principles, the recent tendency has turned toward more-detailed molecularly inspired models. The latter describe the experimentally mapped clock genes and their mutual interactions in the language of chemical kinetics. These models are then solved or simulated using ordinary differential equations or stochastic versions thereof based on the master equation (Gillespie 1977).

Among the most recent models, Goldbeter (1995) proposed a rate equation model to describe the negative feedback of the Period protein in Drosophila melanogaster, including mRNA transcription, translation, and phosphorylation of the protein, as well as nuclear translocation. Subsequent models for circadian clocks in other species can be split somewhat artificially in two groups: (1) models proposing simplified low-dimensional toy models to focus on the role of common network motifs such as nega- tive feedback loops (Barkai and Leibler 2000; Vilar et al. 2002; Gonze and Goldbeter 2006) and (2) more explicit and higher-dimensional models that implement the detailed biochemical processes known to date (Forger and Peskin 2003; Leloup and Goldbeter 2003; Locke et al. 2005). The latter usually contain a large number of unknown rate constants, some of which can be calibrated by imposing experimentally derived constraints such as period length or peak phases under various conditions such as mutant data. The second category of models allows us to make specific predictions (e.g., the influence of the hypophosphorylation of the Per protein in the family advanced sleep phase syndrome [FASPS]; Toh et al. 2001), but such a detailed approach produces a large number of apparently discordant models, each with many unknown parameters, to describe various specific circadian clocks (see discussion in Rand et al. 2006). The first approach, however, focuses on generic properties of cycling systems and seeks to develop an intuitive understanding of the fundamental structures required to explain experimentally observed phenomena such as the apparent loss of rhythmicity in tissue explants. Whereas the low-dimensional models can be fit precisely to experimental data, simulations can be used to relate the explicit biochemical variables of the detailed models to the effective summary variables on the simplified models, thus offering a rather complete view of the problem.

To be ultimately useful to the biologist and not serve merely as toys for theorists, the models should lead to the formulation of testable hypotheses. In Winfree's days, such a prediction was, for example, that there must exist a phase singularity if strong perturbation (type 0 ) phase-resetting is observed and the oscillator has limited cycle properties. He then went on to design an experiment to measure eclosion times in Drosophila that explicitly showed the phase singularity. Nowadays, predictive modeling studies based on rate equation models that are also tested are still rare. 
Synthetic biology seems a good playground for such efforts; compare, e.g., the repressilator system in Escherichia coli (Elowitz and Leibler 2000). Recent studies indicated that modeling the circadian clock can guide identification of new clock components, as in the plant Arabidopsis thaliana (Locke et al. 2005). An often-used methodology to derive predictions is the so-called sensitivity analysis, where model properties are tracked while changing parameters. Typical properties are the period length (Leloup and Goldbeter 2004; Bagheri et al. 2007) or the oscillator stability (Rougemont and Naef 2007).

\section{CLOCK HIERARCHIES, COUPLING, AND ENTRAINMENT}

In higher organisms, timing is organized in a hierarchical manner, with cellular rhythms contributing to rhythmicity of whole organs, and organs being coordinated by a central clock that integrates external inputs such as light or temperature cues. In mammals, the central pacemaker resides in the suprachiasmatic nucleus $(\mathrm{SCN})$ and consists of about 15,000 neurons. This hierarchical mode of transmitting timing information requires mechanisms that can reset or drive the oscillator phases, which is also called entrainment. In addition, cellular oscillators must be accurate enough, a property that can be achieved either by autonomous genetic circuits with sufficient stability properties or alternatively by intercellular coupling as in the SCN. Coupling is known to be effective in the SCN in mammals (Welsh et al. 2004), but its role in peripheral tissues is probably minor (Yoo et al. 2004; Guo et al. 2006; Rougemont and Naef 2007). In essence, stability and coupling are necessary to override noise that is ubiquitous at the cellular level; it is thus the relative balance of stability, coupling, and noise that will determine to what extent specific circadian output functions can be scheduled properly.

\section{BIOLUMINESCENCE}

Long before the availability of modern luciferase reporters, the circadian bioluminescent glow in the unicellular Gonyaulax polyhydra had been exploited to study the underlying properties of the oscillators. In particular, population measurements were modeled mathematically to estimate a high precision of about $1 \%$ in the individual periods (Njus et al. 1981). Importantly, this study emphasized the richness of population signals and proposed a model to extract information such as period dispersion from such signals.

Fluorescence microscopy and bioluminescence recordings have recently allowed the observation of self-sustained oscillators with single-cell resolution. This showed that oscillations are common in individual cells; in fact, they occur by default (Nagoshi et al. 2004; Welsh et al. 2004). Expectedly, the individual oscillators were also noisy, such that their period is not exactly constant but changes from one cycle to the next (Carr and Whitmore 2005). In the absence of intercellular interactions, this leads to desynchronization of the cells, i.e., the phases in a population drift apart until they cancel each other and the population appears globally arrhythmic.

\section{COUPLING}

Several studies explored the possibility of intercellular coupling in peripheral organs and in the SCN. In SCN neurons, coupling depends on synaptic transmission (Liu and Reppert 2000; Yamaguchi et al. 2003; Ohta et al. 2005; Maywood et al. 2006). In the periphery, Guo et al. (2006) have shown that circadian clocks in the liver of SCN-lesioned hamsters lose their phase coherence, whereas Yoo et al. (2004) observed residual oscillations of Per2 expression in various tissue explants for as long as 20 days. It is, however, difficult to determine if these oscillations reflect some intercellular coupling or whether the samples experience partial resetting after the explantation. Liu et al. (2007) have shown that population signals in lung and fibroblast cultures decay faster than in the $\mathrm{SCN}$, for which actual intercellular coupling has been demonstrated, suggesting that there is no or negligible coupling in peripheral tissues.

Because of their high signal-to-noise values, bioluminescence recordings are suited for direct comparison with mathematical models (Izumo et al. 2003; Nagoshi et al. 2004). It is the purpose of this chapter to review a class of models known as phase models and their applications to the analysis of bioluminescence recordings. The focus of this chapter is on the theory and applications of noisyphase models in which the individual oscillator frequencies fluctuate in time while cells are coupled, as in the Kuramoto (1984) model. Analysis of bioluminescence recordings from populations of mouse fibroblasts is considered as an example.

\section{MATHEMATICS OF PHASE MODELS}

Phase oscillators provide an abstraction of a limit-cycle oscillator where one does not worry about the underlying mechanisms generating the oscillations, i.e., the details in the biochemical interactions. Phase oscillators use a parameterization of limit-cycle oscillations in terms of phase and amplitude variables. So in a sense they are much simpler objects than the trajectories for the concentrations of activity levels of genes and proteins. Thus, they provide a coarsegrained description that can be most useful to study generic effects such as fluctuations, oscillator entrainment (forcing) and resetting, and oscillator coupling in cell populations. Phase models have also been popular to discuss synchronization properties in a population of oscillators. (Winfree 1967; Kuramoto 1984; Strogatz 2000; Mihalcescu et al. 2004; Rougemont and Naef 2006). From the Kuramoto study, it was known that gradually increasing the coupling would eventually overcome fluctuations and lead to a macroscopically synchronized state, which is intuitively quite plausible. Synchronyzation here does not mean that all oscillators point exactly in the same phase but that there is a preferred phase orientation or nonuniform phase distribution in the population. The uniformly distributed state is referred to as the incoherent, or desynchronized, state.

\section{The Model}

The fact that circadian clocks trigger oscillating biochemical species is taken as a given. There therefore 
stiff

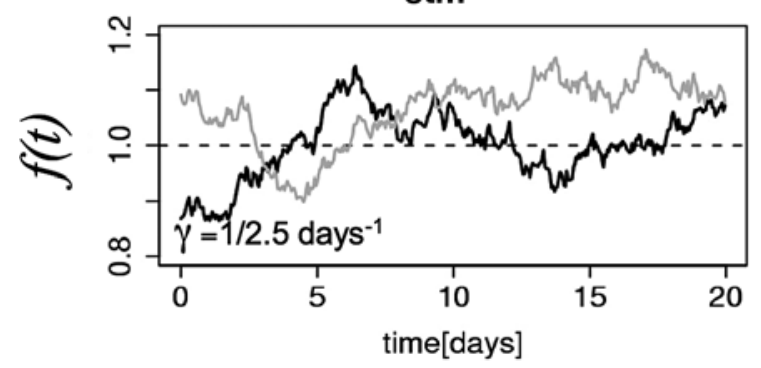

soft

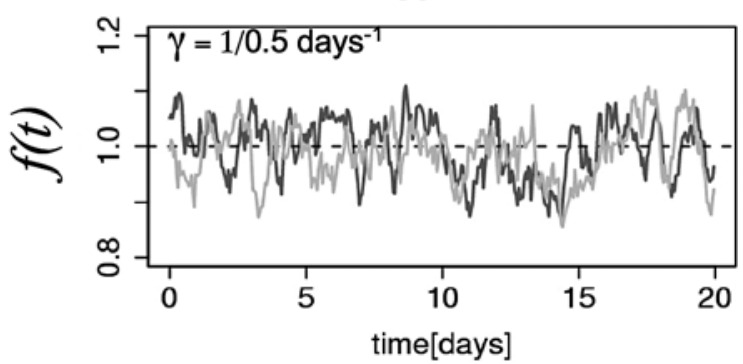

Figure 1. Stiff and soft stochastic frequency trajectories generated according to model in Equation 1 . The variance $\sigma$ (frequency dispersion) is the same for both cases, whereas the mean $\mu=1$.

exists at least two variables that draw a cyclic shape when plotted against each other during one period. This cycle is reparametrized using a phase $(\varphi)$ and a frequency $(f)$ (variations in amplitude are not included in this model). Compared with Kuramoto's original model (Kuramoto 1984) that used static (fixed) frequencies, we generalize to frequencies that can also drift in time. This choice was motivated by experiments in zebra fish cells (Carr and Whitmore 2005) that illustrated how the frequency in individual cells drifts in time. A simple model to represent this possibility is to consider instantaneous frequencies which follow a Gaussian random process that is correlated in time. In other words, the frequencies cannot change too abruptly, and this is controlled by an inverse time constant $\gamma$.

If $\gamma$ is small, the time constant is large and the oscillator is not very stable; on the contrary, very stable oscillators are characterized by short timescales or large $\gamma$. Such a frequency dynamics can be conveniently modeled as an Ornstein-Uhlenbeck process $f(\mathrm{t})$ with the following three parameters:

$$
\mathbf{E}[f(t)]=\mu, \quad \mathbf{E}[(f(t)-\mu)(f(s)-\mu)]=\sigma^{2} e^{-\gamma|t-s|}
$$

where $\mathbf{E}[]$ denotes the expectation. The mean $\mu=1 / 24$ hours sets the circadian periodicity, the rate $\gamma$ is the decay of fluctuations, and the variance $\sigma$ is the amplitude of the fluctuations. Typical trajectories are shown in Figure 1. In independent oscillators, the phase is simply tied to the frequency as:

$$
\frac{d}{d t} \varphi_{i}=f_{i}(t)
$$

The phase dynamics is then also a Gaussian process with mean $\mu t$ and time-dependent variance:

$$
\begin{aligned}
\sigma_{\varphi}^{2}(t) & =\mathbf{E}[(\varphi(t)-\mu t)(\varphi(t)-\mu t)] \\
& =\frac{2 \sigma^{2}}{\gamma^{2}}\left(\gamma t+e^{-\gamma t}-1\right)
\end{aligned}
$$

In these models, the frequencies are coupled to a noise source with variance $\sigma$ that approximates all noise sources in the cellular environment. This is different from the noise simulated in Gillespie-like algorithms, which only take into account state-space-dependent fluctuations due to small number of molecules.

\section{Statistical Properties of Ensembles of Oscillators}

We consider here $N$ individual cells, each described by its own phase and frequency. The phases are initially taken to synchronized by imposing that $\varphi(t=0)=0$. The population signal represented as the average cosine of the phases is an important quantity that can be linked to the data (compare below). When the number of cells $N$ is large and the phases are independent, this average can be related to the time-dependent variance in the phases as follows:

$$
Z_{N}(t)=\frac{1}{N} \sum_{i=1}^{N} \cos \left(2 \pi \varphi_{i}(t)\right) \stackrel{N \rightarrow \infty}{=} \cos (2 \pi \mu t) e^{-\frac{1}{2}\left(2 \pi \sigma_{\varphi}(t)\right)^{2}}
$$

This leads to an oscillation at the mean frequency, with an envelope function represented by an exponential of the variance of the phases. This envelope decays to zero if the variance in the phases keeps increasing, which is the case for nonsynchronized oscillators. Note that if the actual limit cycle is somewhat noncircular, the cosine could be replaced by a more general periodic function. This would in many cases only have a weak effect on the shape of the population average as the higher harmonics have a much larger decay rate in the population average. The population average has two limiting regimes:

$$
e^{-\frac{1}{2}\left(2 \pi \sigma_{\varphi}(t)\right)^{2}} \approx \begin{cases}e^{-(2 \pi \sigma)^{2} t^{2} / 2} & \text { for } t \rightarrow 0 \\ e^{-(2 \pi \sigma / \gamma)^{2}(\gamma t-1)} & \text { for } t \rightarrow \infty\end{cases}
$$

At short times, the population behavior resembles that of static frequencies (indicated by the quadratic time dependence in the exponent), whereas in the long-time limit, we approach a phase diffusion (linear time dependence in the exponent). The dephasing dynamics connecting these two asymptotic formulae provides a way to estimate the relevant parameters from experimental data. In other words, the early decay resembles a bell shape and reflects the frequency dispersion, whereas the longer times reflect phase diffusion.

\section{Interacting Phase Oscillators}

We next introduce coupling among the oscillators. Here, we follow the Kuramoto model and use a simple sine of the phase difference between coupled cells. This introduces an additional parameter $K$ to measure coupling strength: 


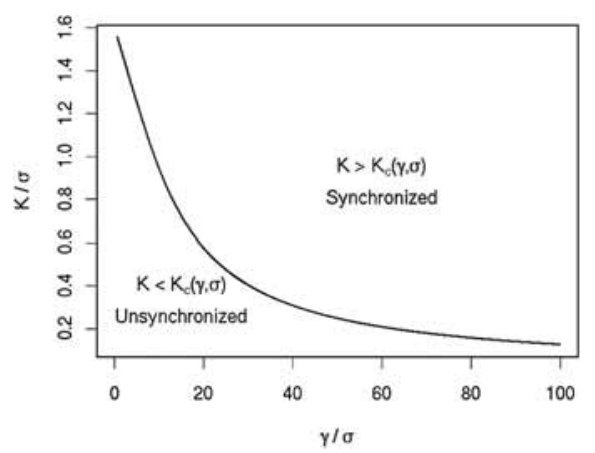

Figure 2. Synchronization thresholds in the coupled phase model. The critical coupling above which the population synchronizes is shown as a function of the damping rate $\gamma$. Coupling and damping are expressed in units of the frequency dispersion $\sigma$. (Adapted, with permission, from Rougemont and Naef 2006 [C American Physical Society].)

$$
\left\{\begin{array}{l}
\frac{d \varphi_{i}(t)}{d(t)}=f_{i}(t)+\frac{K}{\left|N_{i}\right|} \sum_{j \in N_{i}} \sin \left(2 \pi\left(\varphi_{j}(t)-\varphi_{i}(t)\right)\right) \\
\frac{d f_{i}(t)}{d t}=-\gamma\left(f_{i}(t)-\mu\right)+\sqrt{(2 \gamma)} \sigma \eta_{i}(t)
\end{array}\right.
$$

Note that the equation for $f$ is only a convenient alternative description of the Ornstein-Uhlenbeck process introduced above. In this model, cell number $i$ is coupled uniformly to a neighborhood $N_{i}$, and we considered the case of all-to-all coupling for simplicity. The noise source has been reparametrized such that $\eta_{i}(t)$ has unit variance. As in the Kuramoto model, we find a phase transition from a desynchronized state to a collectively synchronized state when the coupling is increased above a threshold $K_{c}$ (Fig. 2) (Rougemont and Naef 2006). Here, synchronization corresponds to the statement that the amplitude of the variable $Z_{N}(t)$ defined above does not tend to zero at large times even for an infinite number of oscillators.

\section{ANALYZING REAL BIOLUMINESCENCE CURVES}

Recent recordings in immortalized (Nagoshi et al. 2004) and primary (Welsh et al. 2004) mouse fibroblasts showed that single cells from peripheral tissues generate cell-autonomous rhythms that could be resynchronized by a serum shock. These single-cell assays were backed up by comparing long (19 days) bioluminescence population recordings with a phase model for static and uncoupled oscillators (Nagoshi et al. 2004). This analysis showed that dephasing, rather than amplitude death, was the dominant cause for amplitude reduction during the 19-day recording. However, the static model underestimated the frequency dispersion. This is expected because the observed frequencies are drifting: Given equal dispersions, drifting frequencies have the property of keeping phase coherence longer, because each oscillator stays out of tune for a shorter average time (see Eq. 2 above).

\section{Envelope Analysis Using Phase Models}

We analyzed a 19-day bioluminescence recording from NIH-3T3 fibroblasts stably transfected with a luceriferase reporter inserted in the Bmall locus (cf. Fig. 3B in Nagoshi et al. 2004) using the above phase model. The data are reproduced here in Figure $3 \mathrm{~A}$. The detrended signal was fit to the prediction for the population average from Equation 3 (Fig. 3B). All parameters could be estimated reliably, and the error bars indicated in Figure $3 \mathrm{C}$ show that the model does not overfit the data. The frequency dispersion was found to be 0.1 days $^{-1}$, which is close to values measured in single cells (Nagoshi et al. 2004). Furthermore, the estimated parameter equals $0.64 \pm 0.17$, reflecting a frequency damping time of 1.56 days and implying that frequency disturbances take longer than a period length to decay. The model could also estimate intercell coupling which indicated that coupling in cell culture might be positive. However, the values estimated were clearly below the synchronization threshold (Fig. 3C). This analysis is detailed in Rougemont and Naef (2007), where it is also substantiated with the analysis of a second bioluminescence curve from Welsh et al. (2004) with similar conclusions.

Recordings in large populations produce signals with a very high signal-to-noise ratio by averaging out noncircadian variations over thousands of individual cells (see Fig. 3) (Welsh et al. 2004). The resulting clean signal has a typical decaying envelope that is a characteristic signature of the underlying dephasing dynamics as shown above. An alternative way of removing random fluctuations can be obtained by averaging the signal produced by a single cell over a large number of periods using the autocorrelation function. We described (Rougemont and Naef 2007) how the autocorrelation and the population average contain similar information and can both be used to fit informative parameters. In practice, however, it is more common to have relatively short recordings (up to 20 periods) of large populations (up to 5000 cells), which is more suitable to the population analysis than the autocorrelation calculations. Furthermore, only supercritical coupling strengths are visible in the autocorrelation; subcritically coupled populations are indistinguishable from uncoupled populations in this type of analysis (Fig. 4).

\section{Coculture Experiment}

Nagoshi et al. (2004) designed a coculture experiment to study the question of intercellular coupling directly. Short-period (20 hours) mutant feeder cells were cocultured with wild-type reporter cells, with a 20 -fold relative excess of nonluminescent feeders. If there were significant coupling, one would predict that the reporter line would slow down its period. Bioluminescence recordings showed no significance phase-shifts over approximately 3 days. To show the potential effect of intercellular coupling in such an experiment, we plotted the expected phase-shift over time for several values of the coupling constant $K$. This is computed as follows:

$$
\begin{aligned}
\delta \varphi(t) & =\varphi_{\text {reporter }}(t)-\frac{t}{24} \\
& =K \int_{0}^{t} \sin \left(2 \pi\left(\varphi_{\text {feeder }}(s)-\varphi_{\text {reporter }}(s)\right)\right) d s \\
& =K \int_{0}^{t} \sin (2 \pi(s(1 / 20-1 / 24)-\delta \varphi(s))) d s
\end{aligned}
$$


A

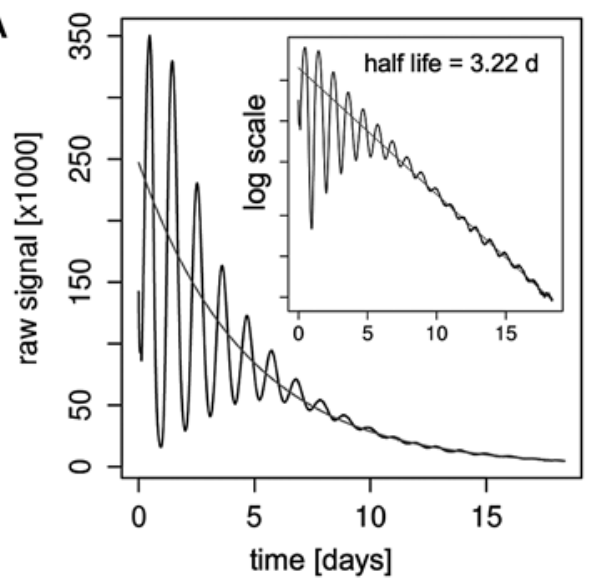

B

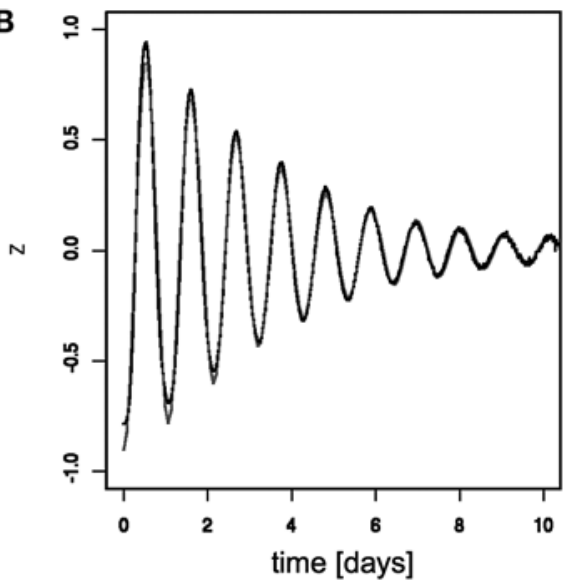

C

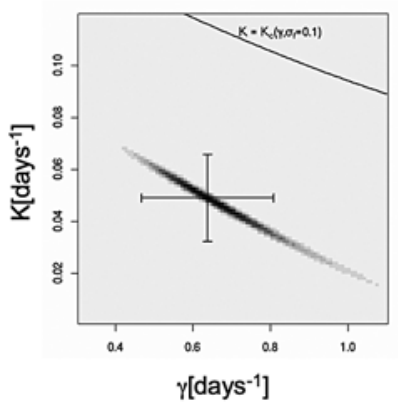

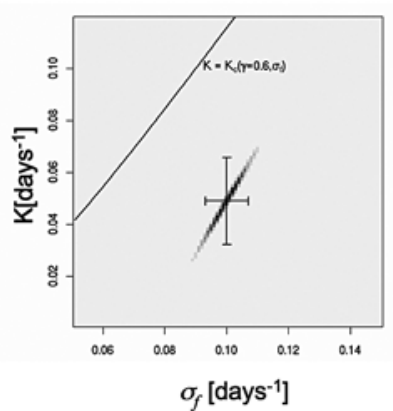

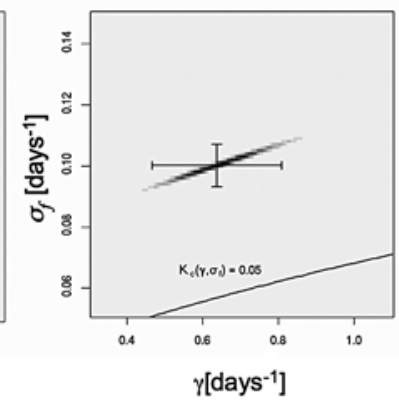

Figure 3. Analysis of population bioluminescence data. (A) Raw bioluminescence counts for a recording of 3 weeks. (Inset) Exponential trend with a half-life of 3.2 days. (B) Detrended signal (black) and the model best fit (gray). (C) Parameter estimates. (Gray shades) Posterior probabilities; (black line) lines of critical coupling. (Reprinted, with permission, from Rougemont and Naef 2007 [Nature Publishing Group].)

First note that the phase-shift does not increase monotonously due to the sine. In the subcritical region, the coupling induces only a slow dephasing that can remain very low for several days (Fig. 5). In particular, a value of $K=$ 0.05 days $^{-1}$ as estimated from population recordings would only shift phases forward by 2.4 hours after 3 days. Note that even a critical coupling of $K=0.1183$ would still require about 10 days to reverse the phase relative to a free-running wild-type cell.

\section{CORRESPONDENCE BETWEEN PHASE AND RATE EQUATION MODELS}

Our analysis has shown that the stability of the frequencies $(\gamma)$ leads to observable effects both in single cells, where it determines the frequency dispersion, and in population recordings of initially synchronized oscillators, where it shapes the long-term decay characteristics of the envelope. One can now ask how is stability determined by the network of coupled chemical reactions that generates the oscillations. The canonical way to discuss the stability of periodic orbits in dynamical systems is through the computation of Floquet multipliers (FMs), which determine the rate at which small perturbations decay back to the limit cycle. These multipliers can be computed from the system of ordinary differential equations (rate models) using computational methods (Doedel et al. 2001). We have explored the Floquet stability in a detailed model of the mammalian clock (Leloup and
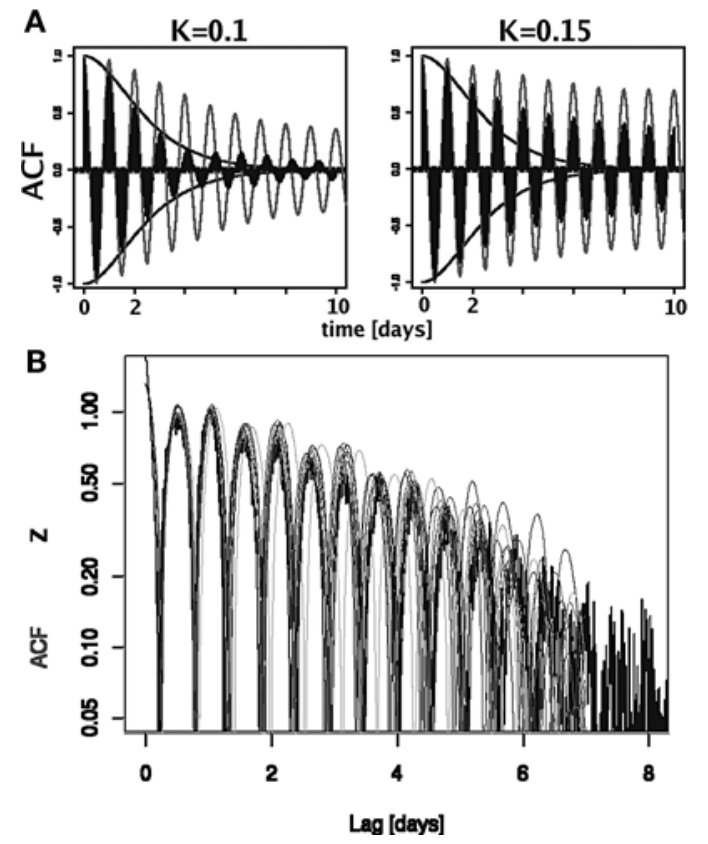

Figure 4. (A) Simulations of 1000 coupled oscillators with coupling strength below and above the synchronization threshold at $K=0.118$. (Black bars) Autocorrelation function of one the oscillators; (black line) population average; (gray line) exact expression for an infinite population of uncoupled oscillators. (B) The population average (thick black bars) is superimposed on the autocorrelations of ten individual cells (gray lines) computed from measurements by Welsh et al. (2004). 


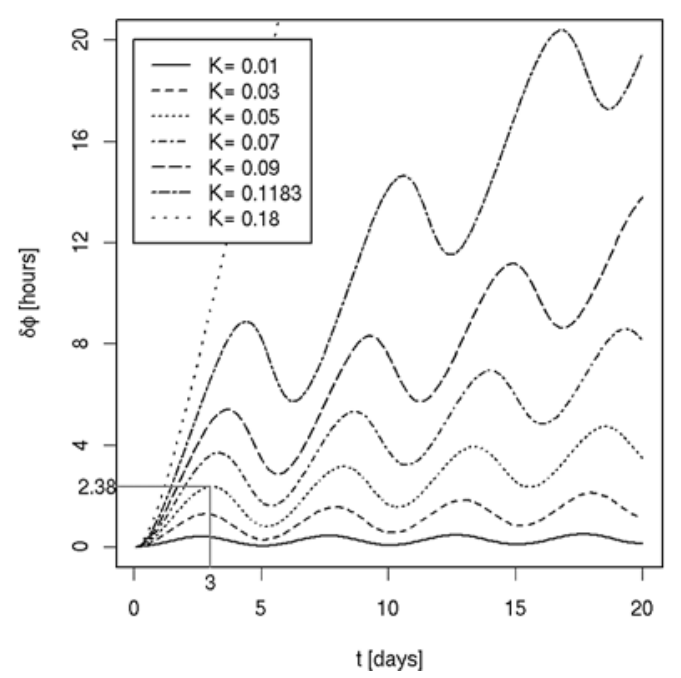

Figure 5. Simulation of the dephasing dynamics in a coculture experiment. The value of $\delta \phi$ was computed numerically over 20 days for several values of $K$. The critical value of $K$ for synchronization is $K=0.1183$. Our estimation from population bioluminescence recordings was $K=0.05$, which leads to a phase-shift of 2.38 hours 3 days after the serum shock.

Goldbeter 2004) by varying single parameters in a window around their nominal values. This computation showed that most parameters have little effects on the stability as measured by FMs, whereas the transcription and phosphorylation rates of the Period gene (only one merged Period gene is included in the model) are among the parameters with the strongest influence (Rougemont and Naef 2007). Such analyses being relevant in the near proximity of the limit cycle, it remains to be investigated to what extent cellular noise sources are small enough such that this regime is indeed probed.

\section{DISCUSSION}

We presented how modeling of noisy-phase oscillators can be fruitful for circadian biology. In the models developed, we introduced the fewest number of parameters necessary to capture generic and experimentally observed phenomena such as drifting frequencies or oscillator coupling. The practical advantage is that the limited parameter set can be determined from currently available data. The downside is that the parameters are not necessarily easy to interpret in molecular terms. For example, explicit coupling mechanisms are not yet known in molecular detail, and we therefore used an effective interaction of phases in terms of a sine function. The molecular basis of stability and its interplay with cellular noise are perhaps a more readily accessible problem. First, we are beginning to obtain relatively detailed kinetic models on the circadian networks generating oscillations, and second, we also have the theoretical methodologies for sensitivity and stability analyses of differential equation models. We also know how to account for cellular fluctuations in these models through the use of stochastic simulation algorithms.

Our analysis of cultured cells showed that intercellular coupling is certainly too weak for synchronizing populations. Our best fits do, however, suggest a small residual coupling. Theoretically, the presence of a positive subcritical coupling may facilitate resynchronization of tissues by systemic cues in the event that the latter would be confined to relatively short-time windows. Although the systems are difficult to compare, a recent report in cyanobacteria used an ingenious mixing of two initially out-of-phase populations to directly measure coupling strength and found an upper bound for the coupling that is about 30 times smaller than our values (Amdaoud et al. 2007). A similar approach could be applied to mammalian cells.

A recent work (Liu et al. 2007) specifically addressed the role of coupling in the SCN compared to peripheral organs such as liver and lung. These authors showed that intercellular coupling is active (and required) in the SCN by showing that SCN explants from Cry 2 mutant mice maintain a synchronized rhythmic expression of Per2, although dissociated neurons rapidly drifted out of phase. In peripheral organs, however, tissue explants displayed a similar decay of the Per2 bioluminescence at the population level than confluent cultures of dissociated fibroblasts. By comparing their bioluminescence recordings of SCN and lung explants (see Fig. 1) (Liu et al. 2007) to those of cultured fibroblasts (see Fig. 2) (Liu et al. 2007), we observed that the envelope of the population signal does not decay to zero in the SNC, whereas the lung explant and the cell culture have a visually similar decay rate, which in the light of our phase models is fully consistent with a high (supercritical) coupling in the SCN and a low (subcritical) coupling in the peripheral organs.

Most importantly, several interesting model systems exhibit molecular oscillations, e.g., the respiratory cycle in yeast or the segmentation clock in vertebrates, for which powerful molecular reporter techniques are being developed. It is thus desirable to have at hand a theoretical framework that allows one not only to extract the most pertinent information from the data, but also to bridge the gap between the often unknown or too complex microscopic description and a phenomenological lower-dimensional description in terms of few highly informative parameters. We believe that phase models do qualify for this purpose and forecast that they will continue to provide useful insights for circadian biologists.

\section{ACKNOWLEDGMENTS}

The authors acknowledge stimulating discussions with Benoit Kornmann and Ueli Schibler. This work was supported by a Swiss National Science Foundation grant to F.N.

\section{REFERENCES}

Amdaoud M., Vallade M., Weiss-Schaber C., and Mihalcescu I. 2007. Cyanobacterial clock, a stable phase oscillator with negligible intercellular coupling. Proc. Natl. Acad. Sci. 104: 7051.

Bagheri N., Stelling J., and Doyle F.J., III. 2007. Quantitative performance metrics for robustness in circadian rhythms. Bioinformatics 23: 358.

Barkai N. and Leibler S. 2000. Circadian clocks limited by noise. Nature 403: 267

Carr A.J. and Whitmore D. 2005. Imaging of single light-responsive clock cells reveals fluctuating free-running periods. Nat. 


\section{PHASE OSCILLATORS AND BIOLUMINESCENCE RECORDINGS}

Cell Biol. 7: 319 .

Doedel E.J., Paffenroth R.C., Chapneys A.R., Fairgrieve T.F., Kutzetsov Y.A., Oldman B.E., Sandstede B., and Wang X.J. 2001. AUTO2000: Continuation and bifurcation software for ordinary differential equations. Technical report, California Institute of Technology, Pasadena, California.

Elowitz M.B. and Leibler S. 2000. A synthetic oscillatory network of transcriptional regulators. Nature 403: 335 .

Forger D.B. and Peskin C.S. 2003. A detailed predictive model of the mammalian circadian clock. Proc. Natl. Acad. Sci. 100: 14806.

Gillespie D.T. 1977. Exact stochastic simulation of coupled chemical reactions. J. Phys. Chem. 81: 2340.

Goldbeter A. 1995. A model for circadian oscillations in the Drosophila period protein (PER). Proc. Biol. Sci. 261: 319.

Gonze D. and Goldbeter A. 2006. Circadian rhythms and molecular noise. Chaos 16: 026110.

Guo H., Brewer J.M., Lehman M.N., and Bittman E.L. 2006. Suprachiasmatic regulation of circadian rhythms of gene expression in hamster peripheral organs: Effects of transplanting the pacemaker. J. Neurosci. 26: 6406.

Izumo M., Johnson C.H., and Yamazaki S. 2003. Circadian gene expression in mammalian fibroblasts revealed by real-time luminescence reporting: Temperature compensation and damping. Proc. Natl. Acad. Sci. 100: 16089.

Kuramoto Y. 1984. Chemical oscillations, waves, and turbulence. Springer-Verlag, Berlin.

Leloup J.C. and Goldbeter A. 2003. Toward a detailed computational model for the mammalian circadian clock. Proc. Natl. Acad. Sci. 100: 7051.

2004. Modeling the mammalian circadian clock: Sensitivity analysis and multiplicity of oscillatory mechanisms. J. Theor. Biol. 230: 541.

Liu A.C., Welsh D.K., Ko C.H., Tran H.G., Zhang E.E., Priest A.A., Buhr E.D., Singer O., Meeker K., Verma I.M., Doyle F.J., III, Takahashi J.S., and Kay S.A. 2007. Intercellular coupling confers robustness against mutations in the SCN circadian clock network. Cell 129: 605.

Liu C. and Reppert S.M. 2000. GABA synchronizes clock cells within the suprachiasmatic circadian clock. Neuron 25: 123.

Locke J.C., Millar A.J., and Turner M.S. 2005. Modelling genetic networks with noisy and varied experimental data: The circadian clock in Arabidopsis thaliana. J. Theor. Biol. 234: 383

Maywood E.S., Reddy A.B., Wong G.K., O’Neill J.S., O’Brien J.A., McMahon D.G., Harmar A.J., Okamura H., and Hastings M.H. 2006. Synchronization and maintenance of timekeeping in suprachiasmatic circadian clock cells by neuropeptidergic signaling. Curr. Biol. 16: 599.

Mihalcescu I., Hsing W., and Leibler S. 2004. Resilient circa- dian oscillator revealed in individual cyanobacteria. Nature 430: 81 .

Nagoshi E., Saini C., Bauer C., Laroche T., Naef F., and Schibler U. 2004. Circadian gene expression in individual fibroblasts: Cell-autonomous and self-sustained oscillators pass time to daughter cells. Cell 119: 693.

Njus D., Gooch V.D., and Hastings J.W. 1981. Precision of the Gonyaulax circadian clock. Cell Biophys. 3: 223.

Ohta H., Yamazaki S., and McMahon D.G. 2005. Constant light desynchronizes mammalian clock neurons. Nat. Neurosci. 8: 267.

Rand D.A., Shulgin B.V., Salazar J.D., and Millar A.J. 2006. Uncovering the design principles of circadian clocks: Mathematical analysis of flexibility and evolutionary goals. $J$. Theor. Biol. 238: 616 .

Rougemont J. and Naef F. 2006. Collective synchronization in populations of globally coupled phase oscillators with drifting frequencies. Phys. Rev. E Stat. Nonlin. Soft Matter Phys. 73: 011104 .

2007. Dynamical signatures of cellular fluctuations and oscillator stability in peripheral circadian clocks. Mol. Syst. Biol. 3: 93.

Strogatz S.H. 2000. Nonlinear dynamics and Chaos: With applications to physics, biology, chemistry and engineering. Perseus Books, Cambridge, Massachusetts.

Toh K.L., Jones C.R., He Y., Eide E.J., Hinz W.A., Virshup D.M., Ptacek L.J., and Fu Y.H. 2001. An hPer2 phosphorylation site mutation in familial advanced sleep phase syndrome. Science 291: 1040.

Vilar J.M., Kueh H.Y., Barkai N., and Leibler S. 2002. Mechanisms of noise-resistance in genetic oscillators. Proc. Natl. Acad. Sci. 99: 5988.

Welsh D.K., Yoo S.H., Liu A.C., Takahashi J.S., and Kay S.A. 2004. Bioluminescence imaging of individual fibroblasts reveals persistent, independently phased circadian rhythms of clock gene expression. Curr. Biol. 14: 2289.

Winfree A.T. 1967. Biological rhythms and the behavior of populations of coupled oscillators. J. Theor. Biol. 16: 15. . 2001. The Geometry of biological time, 2nd edition. Springer, New York.

Yamaguchi S., Isejima H., Matsuo T., Okura R., Yagita K., Kobayashi M., and Okamura H. 2003. Synchronization of cellular clocks in the suprachiasmatic nucleus. Science 302: 1408 .

Yoo S.H., Yamazaki S., Lowrey P.L., Shimomura K., Ko C.H., Buhr E.D., Siepka S.M., Hong H.K., Oh W.J., Yoo O.J., Menaker M., and Takahashi J.S. 2004. PERIOD2::LUCIFERASE real-time reporting of circadian dynamics reveals persistent circadian oscillations in mouse peripheral tissues. Proc. Natl. Acad. Sci. 101: 5339. 


\section{$8_{\text {SYMPOS \& }}^{\infty}$ Cold Spring Harbor Symposia SYMPOSIA on Quantitative Biology}

\section{Stochastic Phase Oscillators and Circadian Bioluminescence Recordings}

J. Rougemont and F. Naef

Cold Spring Harb Symp Quant Biol 2007 72: 405-411

Access the most recent version at doi:10.1101/sqb.2007.72.044

References This article cites 30 articles, 10 of which can be accessed free at: http://symposium.cshlp.org/content/72/405.full.html\#ref-list-1

License

Email Alerting Receive free email alerts when new articles cite this article - sign up in the box at the Service top right corner of the article or click here.

To subscribe to Cold Spring Harbor Symposia on Quantitative Biology go to: http://symposium.cshlp.org/subscriptions 